\title{
Timing pulsars: An exercise in statistical analysis and the scientific process
}

\author{
John R. Walkup ${ }^{1, *}, 2$, Joseph White ${ }^{1}$ and Roger Key ${ }^{1}$ \\ ${ }^{1}$ Department of Physics, California State University, Fresno, Fresno, California, USA and ${ }^{2}$ Present Affiliation: \\ College of Mathematics and Science, University of Central Oklahoma, Edmond, Oklahoma, USA \\ *jwalkup2@uco.edu
}

\begin{abstract}
A lab activity for teaching students the fundamentals of statistical analysis by timing pulsar periods is described. The electromagnetic pulses of pulsars have been mapped to sound and uploaded on social media channels, allowing students to "listen" to the beat of the pulsar. Because these beats are extraordinarily precise, they can serve as cyclic events of known time duration. The three-step process described in this article first requires that students select a timing method of low random error found by comparing standard deviations between two suggested methods. In the second step, students reduce systematic error by calibrating their optimal method using a pulsar of known time duration. Finally, students time an unknown pulsar (the mystery pulsar) using the optimal method chosen in Step 1 and calibrating out the bias found in Step 2. By expressing their results in terms of confidence intervals, they use a professional pulsar database to identify the mystery pulsar. Because students are not informed of the identity of the pulsar until after they turn in their lab reports, they are compelled to perform the measurements as carefully and objectively as possible. This activity provides a perfect vehicle for astronomy labs at the beginning of a semester including online instruction - because it requires no prior instruction in astronomy and no equipment other than the stopwatch on a cell phone and internet connection. Furthermore, this activity offers an introduction to pulsars and such physics topics as magnetism and the conservation of angular momentum.
\end{abstract}

Keywords: Pulsars; Error analysis; Standard deviation; Standard error; Confidence intervals

\section{Introduction}

\subsection{Background}

A recent article described a three-step procedure for teaching students to conduct scientific measurement that reduces random and systematic error (Walkup et al., 2019). That article relied on the use of a light pulse generated by an Arduino. The activity described here uses the same three-step procedure, but replaces the Arduino with the electromagnetic pulse of a pulsar.

The pedagogical purposes of the lab activity discussed here mirrors that in Walkup et al. (2019). For one, this activity at- tempts to supplant the traditional verification lab, where students simply complete stepwise procedures to verify physics concepts learned in lecture, with more career-oriented experimental strategies likely to be used by practicing scientists and engineers. Furthermore, since students in the lab activity described here do not know the values of the physical properties they are measuring until after they turn in their lab reports, the laboratory activity described here helps diminish the confirmation bias and outright fudging that students often employ to enhance the success of their experiment and, therefore, earn a higher grade. 
Table 1. Pulsars available on the author's YouTube channel, with periods omitted from view (Walkup, 2020a,b,c,d,e). The periods are rounded to the nearest 0.001 seconds given that ordinary timing devices available to students only measure to the 0.01 seconds. The sources for each pulsar sound file are also tabulated (Department of Astronomy Cornell University, 2006; Pulsar Central, 2000).

\begin{tabular}{|c|c|c|c|c|}
\hline Label & Pulsar & Period(s) & Link & Source \\
\hline Pulsar A & $\mathrm{B} 2020+28$ & 0.343 & https://youtu.be/sf JDeKSa208 & RadioSky.com \\
\hline Pulsar B & B0329+54 & 0.716 & https://youtu.be/DW108PjTRe4 & RadioSky.com \\
\hline Pulsar C & B0833-45 & 0.089 & https://youtu.be/E7uA5Zn9R1Q & Cornell \\
\hline Pulsar D & B0950+08 & 0.253 & https://youtu.be/2D1RxKT3mxg & RadioSky.com \\
\hline Pulsar E & $\mathrm{B} 1933+16$ & 0.359 & https://youtu.be/HUAltLCuWoM & Cornell \\
\hline
\end{tabular}

\subsection{Pulsars}

Pulsars are the remnants of massive stars that have collapsed under their own weight. The resulting supernovae leave behind neutron stars of immense density but much smaller size. Because the moment of inertia of a neutron star is dwarfed by that of the original star, conservation of angular momentum makes the neutron star spin at incredibly high rates. Spin frequencies range from $0.04 \mathrm{~Hz}$ for PSR J0250+5854 all the way up to 716 $\mathrm{Hz}$ for pulsar PSR J1748-2446ad (Hessels et al., 2006; Tan et al., 2018).

Pulsars emit beams of electromagnetic radiation along their magnetic axes. Because the axis of rotation of a pulsar does not necessarily correspond to its magnetic axis, this radiation functions similarly to a navigation beam for any observer that crosses its path. As such, the pulsar appears to "pulse" in much the same way that a lighthouse appears to pulse to someone on a ship.

Some astronomers have recently mapped the electromagnetic pulses of pulsars to sound and others have loaded these files onto YouTube for easy playing (Department of Astronomy Cornell University, 2006; Pulsar Central, 2000; s7Range, 2018) This allows students to "hear" the pulses of the pulsar with nothing more than an internet connection.

The goal of this lab activity is for students to identity a mystery pulsar by timing its period. Most sources of pulsar sound files also note the true periods of the pulsars, therefore negating their use as a mystery pulsar. In response, one of the authors (Walkup) has uploaded some of the clearer sound files to separate YouTube pages (Walkup, 2020 b,c,d,e). These pulsars are listed in Table 1

\section{Method}

In this laboratory activity, students attempt to identify an unknown (at least to them) pulsar by timing its period, then entering their results into an online database. Although seemingly simple, their ability to obtain a result that exactly matches that of the unknown pulsar is effectively zero because of both random and systematic error. At best, they can only obtain a range of periods for which they can say the unknown pulsar "most likely" or "almost certainly" resides.

\subsection{Reducing random error}

The first step in this activity is to diminish random error by choosing a timing method that produces minimal variation in results. Using two different timing methods, students measure the standard deviation (Eqn.1) in each, where $T_{i}$ represents the $i$ th individual period measurement and $\bar{T}$ represents the average of all $N$ measurements. Students simply choose that method which produces the smallest standard deviation. ${ }^{1}$

1 Strictly speaking, students should use the population standard deviation $\sigma$ rather than the sample standard deviation s. However, since they are

$$
s=\sqrt{\frac{\sum_{i=1}^{N}\left(\bar{T}-T_{i}\right)^{2}}{N-1}}
$$

The laboratory assistant can offer any number of different timing methods to students. For example, students can compare the precision between using a stopwatch and the timer on their cell phones. Because we were forced to teach online, we could not expect students to possess a stopwatch, so we changed the methods to the following:

1. Measure the time between each pulse using 5 trials.

2. Measure the time it takes for 5 pulses to complete, then dividing by 5 .

Students should note that the total time of measurement is the same in both cases; only the manner in which the time is divided differs between the two. In our lab, we relate this time of measurement in terms of observatory cost, that is, both methods will cost the astronomy team the same in terms of money and labour, but one would likely prove more precise than the other. In this sense, this first step offers a glimpse into datadriven decision making.

\subsection{Reducing systematic error}

In Step 2, students use the optimal method found in Step 1 to measure the period of a pulsar with a disclosed period. By comparing their sample mean to that of the known period, they determine the bias in their optimal method. This bias allows them to correct for systematic error through calibration. For example, if students obtained a period of 0.52 seconds for a pulsar of known duration 0.50 seconds, then they know to calibrate any future results by subtracting 0.02 seconds, in principle eliminating the systematic error in their measurements.

\subsection{Estimating unknowns}

In Step 3, students use the optimal method found in Step 1 to time the period of a mystery pulsar, correcting for the bias found in Step 2. They also calculate the standard error

$$
S E=\frac{S}{N}
$$

in their results by hand or using a spreadsheet. They then translate this standard error into the language of confidence intervals straddling the sample mean of their results. For students new to statistics, we have found the following explanations helpful:

only comparing one standard deviation with the other, such a distinction serves no practical purpose. 
1. The period of the unknown pulsar most likely ranges between $T_{\text {low }}$ to $T_{\text {high }}$, where $T_{\text {low }}$ and $T_{\text {high }}$ represent 1 SE above and below the sample mean, respectively. Note that "most likely" corresponds to roughly a $68 \%$ likelihood.

2. The period of the unknown pulsar almost certainly ranges between Tlow to Thigh, where $T_{\text {low }}$ and $T_{\text {high }}$ represent $2 S E$ above and below the sample mean, respectively, where "almost certainly" corresponds to roughly a 95\% likelihood.

Students then enter these ranges into the search tool provided by the ATNF Pulsar Catalogue using the variable PO, which represents the barycentric period of the pulsar (Manchester et al., 2005; Australia Telescope National Facility, 2020). The database then generates a list of pulsars that fall within these ranges. These pulsars serve as "likely culprits" for the mystery pulsar.

For example, students that obtain an average pulse of 0.044 seconds with a standard error of 0.001 seconds can assume the period of the mystery pulsar "most likely" falls between 0.043 and 0.045 seconds and "almost certainly" falls between 0.042 and 0.046 seconds $^{2}$. Inserting the command "PO $>0.043 \& \&$ $\mathrm{PO}<0.045$ " into the text field titled "Condition" generates the three pulsar candidates in Table 2 . Using a range generated by $\pm 2 \mathrm{SE}$, we can generate the larger number of pulsars in Table 3 for which the unknown pulsar almost certainly is listed (assuming that students have effectively calibrated away systematic error in Step 2).

\section{Results}

A total of 33 students enrolled in a calculus-based introductory physics laboratory course completed the pulsar activity during the Northern Hemisphere Spring 2020 semester. The authors omitted 8 students from consideration because they displayed no meaningful effort to complete the activity, leaving 25 students in the sample. Of these, 5 were able to produce a list of pulsars containing the identity of the mystery pulsar.

Gauging success proved difficult. For example, a few students (5) failed to provide a list containing the mystery pulsar, but largely because their confidence intervals were so small that all but the tiniest bias pushed their range of periods too far from the true period. In this sense, the more careful they conducted Step 1, the less likely they would find suitable candidates for the mystery pulsar. Alternatively, sloppy timing would produce such large confidence intervals that a student's chances of producing a list containing the mystery pulsar would grow. As such, we graded the students' lab reports holistically based on their ability to generate reasonably small confidence intervals and reduce bias.

The number of pulsars students listed in the $68 \%$ confidence interval ranged from 11 to 17 , with 21 to 30 in the $95 \%$ confidence interval ${ }^{3}$. Ten students relied on a mean period that was so far from the true that any meaningful reflection on their results would have uncovered an obvious mistake. with significant differences that could have been easily found (e.g.: it was easy to see that the measured period was way too large).
Table 2. Pulsars generated by the ATNF Pulsar Catalogue with periods ranging \pm 1 SE about the sample mean of 0.044 seconds, assuming $\mathrm{SE}=0.001$ seconds.

\begin{tabular}{llll}
\hline No. & Pulsar & Frequency $(\mathrm{Hz})$ & Period (s) \\
\hline 1 & Jo557-2948 & 22.91337153767 & 0.0436426389 \\
2 & J1157-5112 & 22.94144832067 & 0.04358922706 \\
3 & J1813-1749 & 22.37171236 & 0.04469930526 \\
\hline
\end{tabular}

Table 3. Same as Table 2, but using a confidence interval of \pm 2 SE.

\begin{tabular}{llll}
\hline No. & Pulsar & Frequency $(\mathrm{Hz})$ & Period $(\mathrm{s})$ \\
\hline 1 & J0453+1559 & 21.8427329517106 & 0.04578181687 \\
2 & J0557-2948 & 22.91337153767 & 0.0436426389 \\
3 & J1157-5112 & 22.94144832067 & 0.04358922706 \\
4 & J1813-1749 & 22.37171236 & 0.04469930526 \\
5 & J0453+1559 & 21.8427329517106 & 0.04578181687 \\
6 & J1454-5846 & 22.10004678013 & 0.045248773 \\
\hline
\end{tabular}

\section{Discussion}

Although a challenge, results show that with enough care students can generate a reasonably small number of pulsars containing the identity of the mystery pulsar. This activity does not involve any more knowledge of astronomy than the lab instructor desires. As such, it poses a useful lab activity for the first few weeks of instruction and addresses many concepts in error analysis that students will need for the remainder of the semester. For example, this activity provides useful dialog in terms of significant figures, as students will try to input ridiculously precise values into the database. Typically, students fail to appreciate the importance of significant figures and treat them simply as a requirement they must satisfy so as to not lose scoring credit. In this activity, paying heed to significant figures changes the range of periods they submit to the ATNF database and therefore the number of pulsars the database will offer.

This activity also clarifies the difference between the standard deviation from the standard error. Students only use the standard deviation in Step 1 because at that point they only care which of the two methods produces results with the least variation. They rely on the standard error in Step 3 because they need an estimation of how far their sample mean will range from the true mean. This activity also clearly delineates random from systematic error because each type of error is examined in its own step of the process. Furthermore, the impact of random error and systematic clarify: Systematic error pushes the confidence interval away from the true sample mean. This means that their list might not include the mystery pulsar at all.

A large random error, however, generates a list too large. While the mystery pulsar may be listed in the results, the huge number of listed pulsars makes the results worthless. These considerations post challenges for any objective attempt to grade lab reports simply on whether students included a list with the mystery pulsar in it. We suggest holistically grading students' results based on two considerations: (a) Does the size of their confidence interval indicate careless timing in Step 1 and (b) does the bias in their results indicate substandard calibration in Step 2?

\section{References}

2 Out of concerns for brevity, we are using a relatively short pulse as an example in this article because there are relatively few pulsars that have such periods. For the same reason, we chose a standard error more precise than the students can obtain with a simple stop watch. As such, students will generate much a much larger list of pulsars than shown here.

3 One student only listed one pulsar, the correct one. We consider this result an anomaly.
Australia Telescope National Facility (2020). The ATNF Pulsar Database. Retrived from https://www.atnf.csiro.au/ research/pulsar/psrcat/.

Department of Astronomy Cornell University (2006). Pulsar 
sounds. Retrived from http://hosting.astro.cornell.edu/ $\sim$ deneva/psr_sounds/pulsars_sounds.htm.

Hessels, J. W., Ransom, S. M., Stairs, I. H., Freire, P. C., Kaspi, V. M., and Camilo, F. (2006). A radio pulsar spinning at $716 \mathrm{hz}$. Science, 311(5769):1901-904. https://doi.org/10.1126/ science. 1123430

Manchester, R. N., Hobbs, G. B., Teoh, A., and Hobbs, M. A. (2005). The Australia Telescope National Facility Pulsar Catalogue. The Astronomical Journal, 129(4):1993. https://doi .org/10. $1086 / 428488$.

Pulsar Central (2000). Retrived from http://www.radiosky.com/ rspplsr.html.

s7Range (2018). Pulsar sounds. Retrived from https://youtu. be/35SbvnYEc9c.

Tan, C. M., Bassa, C. G., Cooper, S., Dijkema, T. J., Esposito, P., Hessels, J. W. T., Kondratiev, V. I., Kramer, M., Michilli, D., Sanidas, S., and et al. (2018). Lofar discovery of a 23.5 s radio pulsar. The Astrophysical Journal, 866(1):54. https://doi.org/10.3847/ 1538-4357/aade88.

Walkup, J. R. (2020a). Pulsar A. Retrived from https: //youtu.be/ sf JDeKSa208.

Walkup, J. R. (2020b). Pulsar B. Retrived from https://youtu. be/DW108PjTRe4.

Walkup, J. R. (2020c). Pulsar C. Retrived from https: //youtu. be/ E7uA5Zn9RIQ.

Walkup, J. R. (2020d). Pulsar D. Retrived from https://youtu be/2D1RxKT3mxg.

Walkup, J. R. (2020e). Pulsar E. Retrived from https: //youtu.be/ HUAltLCuWoM.

Walkup, J. R., Key, R. A., and Talbot, P. R. (2019). Timing a light pulse in the introductory physics lab. Physics Education, 54(4):045007. https://doi .org/10.1088/1361-6552/ab15b4. 